Mit Blick auf die Zielgruppen sowohl der Studierenden als auch der Fachkräfte der Sozialen Arbeit und Sozialverwaltung hatte ich in UJ in den Jahren 2014 bis 2017 in acht Folgen einen Überblick über aktuelle Literatur zu unterschiedlichen Rechtsgebieten im Bereich der Sozialen Arbeit vorgelegt, u. a. zum Familienrecht (in UJ 3/2017, 136 - 142) und zum Kinder- und Jugendhilferecht (in UJ 3/2017, 386-392). Nach vier Jahren erscheint ein erneuter Überblick über aktuelle Lehrbücher zum Familienrecht (siehe bereits UJ 7 +8/2021, 338-346) und zum Kinder- und Jugendhilferecht, auch wenn dabei das Kinder- und Jugendstärkungsgesetz (KJSG) noch nicht berücksichtigt werden konnte.

\title{
Lehrbücher zum Kinder- und Jugendhilferecht für die Soziale Arbeit
}

\author{
Rezensent:
}

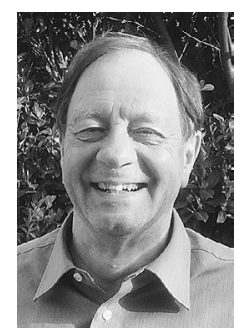

\author{
Prof. Dr. jur. Dr. phil. \\ Reinhard Joachim Wabnitz \\ Jg. 1952; Assessor jur., \\ Magister rer. publ., Ministe- \\ rialdirektor a.D., Professor \\ für Rechtswissenschaft, \\ insbesondere für Familien- \\ und Kinder-und Jugendhilfe- \\ recht, an der Hochschule \\ RheinMain, Wiesbaden
}

Das Kinder- und Jugendhilferecht, insbesondere nach seinem wichtigsten Gesetz, dem Achten Buch Sozialgesetzbuch (Kinder- und Jugendhilfe) - im Folgenden: SGB VIII - ist bekanntlich eines der Kernfächer in den Ausbildungsgängen für Soziale Arbeit, insbesondere in den Bachelorstudiengängen an Hochschulen, Fachhochschulen und Universitäten. Kenntnisse des Kinder- und Jugendhilferechts nach dem SGB VIII (ergänzt durch das jeweils einschlägige Landesrecht, auf das hier wegen sei- ner Unterschiedlichkeit nicht eingegangen wird) sind unverzichtbar für eine erfolgreiche praktische Tätigkeit in allen Arbeitsfeldern der Kinder- und Jugendhilfe, aber auch im Bereich der Familiengerichtsbarkeit und vielfach im Bereich etwa von SGB II (Grundsicherung), III (Arbeitsförderung) oder XII (Sozialhilfe).

Die im Folgenden vorzustellenden Werke richten sich ganz überwiegend explizit an Studierende und PraktikerInnen im Bereich der Sozialen Arbeit, die zugleich Kenntnisse des Familienrechts nach dem 4. Buch des Bürgerlichen Gesetzbuches (Familienrecht) erwerben sollten, da beide Rechtsgebiete - Kinder- und Jugendhilferecht und Familienrecht (siehe dazu UJ 7+8/2021, 338-346) - vielfach aufeinander bezogen und miteinander verwoben sind.

Das Kinder- und Jugendhilferecht ist auch (knapper) Bestandteil der beiden folgenden, breiter angelegten Lehrbücher: 


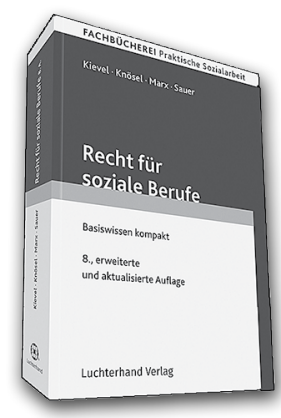

Kievel, W., Knösel, $P$., Marx, A., Sauer, J. (2018):

Recht für soziale Berufe. Basiswissen kompakt

8. Aufl. Wolters Kluwer, Köln. 638 Seiten, ISBN 978-3-472095057, € 39,-

davon allerdings lediglich 42 Seiten zum Kinder- und Jugendhilferecht (von Knösel)

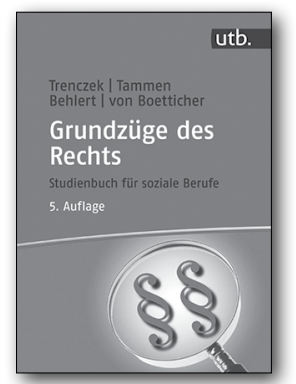

Trenczek, T., Tammen, B., Behlert, W., Boetticher, A.von (2018):

Grundzüge des Rechts. Studienbuch für soziale Berufe

5. Aufl. Ernst Reinhardt, München/Basel. 873 Seiten, ISBN 978-3-8252-8726-9, $€ 49,99$

davon allerdings lediglich 54 Seiten zum Kinder-und Jugendhilferecht (von Tammen/Trenczek)

Bei der Vorstellung der folgenden Lehrbücher zum Kinder- und Jugendhilferecht werde ich jeweils eingehen auf: Autorinnen und Autoren, wesentliche Inhalte der jeweiligen Werke sowie auf deren Zielsetzungen und Adressatenkreise.

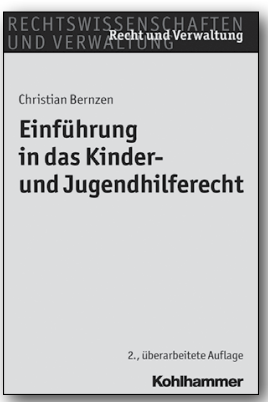

Bernzen, C. (2016):

Einführung in das Kinderund Jugendhilferecht

2. Aufl. Kohlhammer,

Stuttgart. 137 Seiten

ISBN 978-3-17-030022-4, $€ 22,-$

Autor dieses Werkes ist Prof. Dr. Christian Bernzen,

Hochschullehrer an der Katholischen Hochschule für Sozialwesen, Berlin, sowie Rechtsanwalt und Partner von Bernzen Sonntag Rechtsanwälte Steuerberater, Hamburg. Das Werk ist in die folgenden 14 Kapitel, entsprechend dem üblicherweise an den Hochschulen pro Semester zur Verfügung stehenden Zeitkontingent und im Wesentlichen der Kapitel- und Paragrafenfolge des SGB VIII folgend, gegliedert: Einführung in das Rechtsgebiet; Die Allgemeinen Bestimmungen des SGB VIII; Leistungen der Kinder- und Jugendhilfe - mit sechs Kapiteln; Andere Aufgaben; Datenschutz und Statistik; Öffentliche Träger; Freie Träger; Zentrale Aufgaben; Zuständigkeit, Kostenerstattung; Kostenbeteiligungen. Angereichert wird die lehrbuchmäßige Textdarstellung durch 14 einprägsame Fälle und Lösungen und die Präsentation eines Urteils des Bundesverwaltungsgerichts im Wortlaut, aufgrund dessen man sich sehr gut in Stil und Inhalte eines solchen höchstrichterlichen Judikates „einfühlen“ kann.

Das „Einführungsbuch“ will (siehe Seite 1) „in das Rechtsgebiet praxisorientiert einführen" und ist maßgeblich durch die Lehrtätigkeit des Autors inspiriert (siehe Seite 2). Gedacht ist es für die Studierenden, nicht nur zur Klausurvorbereitung, sowie alle sonstigen Nutzerinnen und Nutzer, die sich in der Praxis erstmalig oder erneut mit diesem Rechtsgebiet befassen.

\begin{tabular}{|c|c|}
\hline 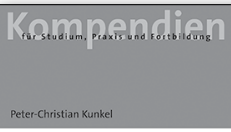 & $\begin{array}{l}\text { Kunkel, P.-C. (2018): } \\
\text { Jugendhilferecht } \\
\text { 9. Aufl. Nomos, Baden- }\end{array}$ \\
\hline Jugendhilferecht & $\begin{array}{l}\text { Baden. } 513 \text { Seiten, } \\
\text { ISBN 978-3-8487-2233-4, } \\
€ 25,- \\
\text { (10. Aufl. vorgesehen für } \\
\text { September 2021) }\end{array}$ \\
\hline X Nomos & \\
\hline
\end{tabular}
erschienenen Werkes ist Peter-Christian Kunkel, ehemals Professor an der Hochschule für öffentliche Verwaltung Kehl und Verwaltungsbeamter in Rheinland-Pfalz.

Das voluminöse Buch ist in sechs große Teile gegliedert: Die Entwicklung des Jugendhilferechts; Grundsätze des Jugendhilferechts; Die Tätigkeitsfelder der Jugendhilfe; Die Organisa- 
tion der Jugendhilfe; Das Verfahren der Jugendhilfe; Die Kosten der Jugendhilfe. Der gut lesbare, auch historisch fundierte, umfangreiche Text wird durch zahlreiche Übersichten und Schaubilder aufgelockert und übersichtlich präsentiert; Letzteres ist geradezu „ein Markenzeichen von Kunkel“. Ergänzt wird der eigentliche Lehrbuchteil des Buches (im Umfang von ca. 300 Druckseiten) durch zahlreiche Anhänge (im Umfang von weiteren ca. 200 Druckseiten: u.a. mit weiteren Schaubildern und Schemata, Rechtsquellenübersichten, Verwaltungsvorschriften, Mustersatzungen, Nomenklaturen, Rechtsprechungsübersicht und Übersichten über weitere Arbeitsmaterialien, Arbeitshilfen und Adressen sowie ein systematisches Literaturverzeichnis).

Das Werk wendet sich zwar auch an Studierende, ist allerdings allein schon wegen seines Umfangs und Detaillierungsgrades mehr ein „Klassiker für Praktiker" (so zu Recht: Poschmann in einer Rezension in FamRZ 2016, 1644). Denen ist es, auch wegen der vielfältigen praxisorientierten und profunden Handreichungen, besonders nachdrücklich zu empfehlen: insbesondere Mitarbeiterinnen und Mitarbeitern von Trägern der freien und öffentlichen Jugendhilfe und Behörden, RechtsanwältInnen und Gerichten.

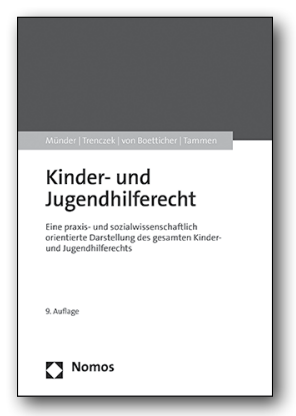

Münder, J., Trenczek, T., Boetticher, A. von, Tammen, B. (2020): Kinder- und Jugendhilferecht. Eine praxis- und sozialwissenschaftlich orientierte Darstellung des gesamten Kinderund Jugendhilferechts

9. Aufl. Nomos, Baden-Baden. 403 Seiten, ISBN 978-3-8487-6595-9, € 24,-

Prof. Dr. Johannes Münder war über Jahrzehnte hinweg an der Technischen Universität Berlin, Lehrstuhl für Sozialrecht und Zivilrecht, tätig. Er hat das Werk vor vielen Jahren begründet. Mit der 7. Aufl. 2011 war Prof. Dr. Thomas Trenczek, M. A., Professor für Straf- und Sozialrecht mit Schwerpunkt Jugend an der Ernst-Abbe-Hochschule Jena, als Co-Autor hinzugetreten, und nunmehr mit der 9. Aufl. Prof. Dr. Arne von Boetticher, Professor an der Fachhochschule Potsdam, sowie Professorin Dr. Britta Tammen, Fachhochschule Neubrandenburg. Das Werk wird nunmehr vom Nomos Verlag, Baden-Baden, herausgegeben (früher: Wolters Kluwer/ Luchterhand).

Auch dieses Werk ist ein „Klassiker" in der Reihe der kinder- und jugendhilferechtlichen Literatur für die Soziale Arbeit. Es ist (insoweit unverändert) in sechs Teile gegliedert - mit 19 fortlaufenden Kapiteln auf der nächsten Gliederungsebene:VorTeil 1:Einführung; 1.Teil:Grundlegung; 2. Teil: Leistungen der Jugendhilfe; 3. Teil: Andere Aufgaben der Jugendhilfe; 4. Teil: Sozialdatenschutz; 5. Teil: Leistungsverpflichtung und -erbringung, Aufgabenverpflichtung und -wahrnehmung sowie deren Finanzierung; 6. Teil: Kinder- und Jugendhilfe im sozial- und gesellschaftspolitischen Kontext.

Die Autorin sowie die Autoren stellen das Kinder- und Jugendhilferecht insbesondere nach dem SGB VIII, aber auch nach anderen zivil- und öffentlich-rechtlichen Gesetzen des Kinder-, Jugend- und Familienrechts, umfassend dar. Rechtsprechung und Literatur werden detailliert einbezogen. Das „Alleinstellungsmerkmal“ des Werkes ist allerdings nach wie vor, dass es sich - siehe Untertitel - (auch) um „eine sozialwissenschaftlich orientierte Darstellung" des Kinderund Jugendhilferechts handelt: Inhaltlich findet eine intensive Einbeziehung sozialwissenschaftlicher Erkenntnisse statt, nicht zuletzt deswegen, weil im Kinder- und Jugendhilferecht - wie im Familienrecht - sozialwissenschaftliches Wissen für die Auslegung und Anwendung des Rechts von besonderer Bedeutung ist (vgl. Vorwort, 1).

Der Umfang des Werkes ist gegenüber den Vorauflagen erheblich ausgeweitet worden: von 252 Seiten (7. Aufl.) auf nunmehr 402 Seiten; 
statt früher in 16 ist es nunmehr in 19 Kapitel untergliedert. Das Buch ist gleichwohl nach wie vor als Lehrbuch konzipiert; es wendet sich an alle, die mit dem Kinder- und Jugendhilferecht zu tun haben, seien es Studierende der Sozialen Arbeit sowie der Rechtswissenschaft als auch Praktikerinnen und Praktiker in diesen Bereichen (siehe Vorwort, 5).

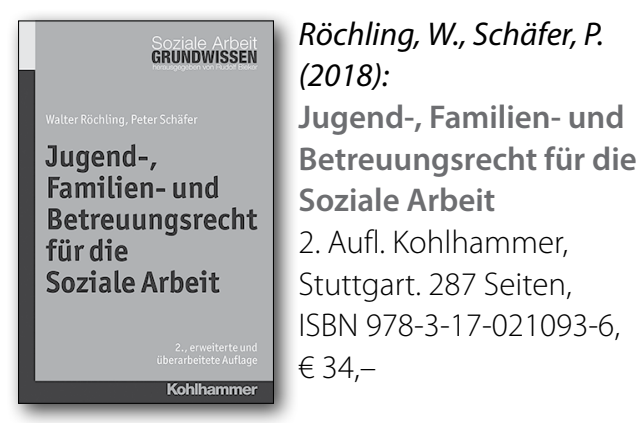

Prof. Dr. Walter Röchling ist Familienrichter i. R. und Honorarprofessor an der Hochschule Niederrhein. Hinzugetreten ist mit der 2. Auflage Prof. Dr. Peter Schäfer, hauptamtlicher Professor an der Hochschule Niederrhein, der vor allem den neuen Teil E. (Mediation) geschrieben hat (251-278).

Das Lehrbuch ist in fünf Teile untergliedert: A. Jugendhilferecht/SGB VIII, B. Familienrecht, C. Betreuungsrecht, D. Verfahrensrechtliche Regelungen sowie E. Mediation - Rechtliche Grundlagen, mediationsfördernde Regelungen im familiengerichtlichen Verfahren und Rechtsdienstleistungsgesetz. Unterhalb der Gliederungsebene der fünf genannten Teile ist das Werk durchlaufend in nunmehr 19 Kapitel (I. bis XIX.) untergliedert. Die einzelnen Kapitel wiederum sind übersichtlich aufgebaut und beinhalten zumeist knappe Einführungen („Was Sie in diesem Kapitel lernen können“), den Text der besonders relevanten Gesetzesbestimmungen im Wortlaut und in grau abgesetzter Kastenform, lehrbuchartige Darstellungen mit unterschiedlichen Detaillierungsgraden sowie prägnante Schlussbemerkungen („Gut zu wissen - gut zu merken").
Schwerpunkt des Werkes mit über 220 Druckseiten sind die Ausführungen in Teil B. bis $E$. zum Familienrecht mit Ausführungen zum Kindschaftsrecht und Adoptionsrecht sowie zu den Themenfeldern Beistandschaft, Vormundschaft, Pflegschaft und Rechtliche Betreuung. Mit Blick darauf habe ich das Werk (in UJ 7+8/2021, 341f) grundsätzlich positiv besprochen, auch wenn es keine Ausführungen zum Eherecht, Ehescheidungsrecht, zum Unterhaltsrecht (sowohl zwischen Ehegatten als auch zwischen Verwandten) sowie zum Recht der nichtehelichen Lebensgemeinschaften einschließlich der Eingetragenen Lebenspartnerschaft enthält.

Die Ausführungen in Teil A. Jugendhilferecht/ SGB VIII fallen mit 35 Seiten demgegenüber mehr als knapp aus und beinhalten lediglich kurze Unterkapitel zu: Kurzüberblick über Grundsätze und Inhalte des Jugendhilferechts; Gewährung von Hilfe zur Erziehung - §27 SGB VIII; Schutzauftrag bei Kindeswohlgefährdung gem. §8 a SGB VIII sowie Inobhutnahme gem. §42 SGB VIII. Damit wird nur ein sehr kleiner Ausschnitt aus dem breiten Spektrum des Kinder- und Jugendhilferechts behandelt. Es fehlen Darstellungen zu den allermeisten Kapiteln und der ganz überwiegenden Zahl der ca. 150 Vorschriften des SGB VIII.

Das Buch wendet sich ausdrücklich an Studierende der Sozialen Arbeit, denen es ermöglichen soll, „sich den Lernstoff im Bereich des Jugend-, Familien- und Betreuungsrechts selbstständig oder als Begleitung zu den entsprechenden Lehrveranstaltungen anzueignen"; die Schwerpunkte des Werkes sind jedoch auch „an der praktischen Tätigkeit von Sozialarbeitern ausgerichtet" (siehe Umschlagsrückseite). Das Werk kann zur Vorbereitung auf Lehrveranstaltungen und Prüfungen zum Familienrecht empfohlen werden; aus den genannten Gründen jedoch nicht mit Blick auf solche zum Kinder- und Jugendhilferecht. 


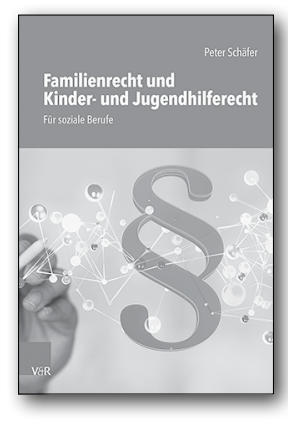

Schäfer, P. (2021):

Familienrecht und Kinderund Jugendhilferecht

für soziale Berufe.

Grundlagen und exemplarische Vertiefungen

Vandenhoeck \& Ruprecht,

Göttingen. ISBN 978-3-525-

71777-6, € 22,-

(vorgesehenes Erscheinen:

März 2022)

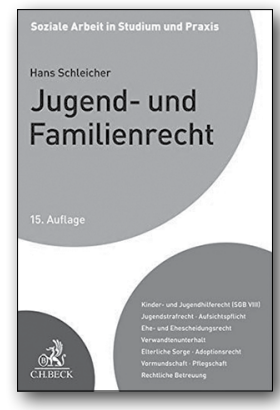

Schleicher, H. (2020):

Jugend- und Familien-

recht. Ein Studienbuch

15. Aufl. Beck, München.

407 Seiten, ISBN 978-3-406-

$74579-9, € 29,80$

Der kürzlich leider verstorbene Begründer des Wer-

kes, Prof. Hans Schleicher, war viele Jahre Professor an der Hochschule München. Wenn ich es richtig sehe, handelt es sich bei seinem bereits 1973 in 1. Aufl. erschienenen Buch um das erste Werk dieser Art, das gezielt für Studierende und PraktikerInnen der Sozialen Arbeit verfasst worden ist. Und kein anderes der hier angezeigten Werke speziell für diesen Adressatenkreis hat ebenfalls bereits eine 15. Aufl. erlebt! Autorin und Autoren sind nunmehr Rechtsanwalt Dieter Küppers, München, Prof. Dr. Annette Rabe, München, Prof. Dr. Jürgen Winkler, Freiburg, sowie Dr. Sebastian Wußler, Direktor des Amtsgerichts Bühl.

In dem genannten Buch werden sowohl das "Jugendrecht" (Teil 1) als auch das "Familienrecht" (Teil 2) dargestellt; zu Letzterem habe ich mich bereits in UJ 7+8/2021 (342) positiv geäußert. Teil 1 enthält die für beide Bereiche grundlegenden Kapitel 1 (Rechtliche Bedeutung der einzelnen Altersstufen) und 2 (Aufsichtspflicht im privaten und beruflichen Alltag). Kapitel 3 (Kinder- und Jugendhilferecht - mit knapp 100 Druckseiten) beinhaltet nach allgemeinen Hinweisen (A.) die
Darstellung der Grundsätze des SGB VIII (B.), der Jugendhilfeleistungen (C.), der "Anderen“ Aufgaben (D.) sowie des Datenschutzes (E.). Daran schließt sich Kapitel 4 mit Ausführungen zum Jugendstrafrecht an (ca. 55 Seiten).

Das Werk ist sehr übersichtlich gegliedert - und erschließt sich nicht zuletzt auch deshalb besonders leicht, weil bei allen Absätzen links oder rechts am Rand jeweils ein Stichwort ausgebracht ist, das den jeweiligen Inhalt kurz und knapp "auf den Punkt bringt". Dies stellt durchaus ein "Alleinstellungsmerkmal“ dar!

Auf der Umschlagsrückseite heißt es zu diesem Werk: „Das Studienbuch wendet sich vor allem an Studierende und Praktiker der Sozialarbeit/Sozialpädagogik. Es vermittelt fundierte Kenntnisse wesentlicher Gebiete des Jugend- und Familienrechts und deckt die für die Ausbildung und Praxis wichtigsten Bereiche ab. Es ist aber auch als Hilfe für diejenigen gedacht, die sich sonst mit dem Jugend- und Familienrecht befassen."

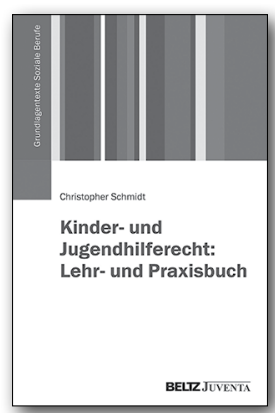

Schmidt, C. (2017):

Kinder- und

Jugendhilferecht.

Lehr- und Praxisbuch

Beltz Juventa, Weinheim/

Basel. 223 Seiten gebunden, ISBN 978-3-7799-2363-3,

$€ 16,95$

Im Jahre 2016 hatte Christopher Schmidt, Professor an der Hochschule Esslingen, im Kohlhammer Verlag sein "Parallelbuch": Familienrecht und Einführung in das Zivilrecht. Lehr-und Praxisbuch für die Kinder-und Jugendhilfe vorgelegt. Es folgte das hier anzuzeigende weitere Werk zum Kinder- und Jugendhilferecht bei Beltz Juventa, in dem die Grundzüge des Kinder- und Jugendhilferechts aufgezeigt werden (Vorwort, 5).

Das Buch ist, im Wesentlichen entsprechend der Kapitel- und Paragrafenfolge des Achten 
Buches Sozialgesetzbuch (SGB VIII - Kinder- und Jugendhilfe), in XIX Kapitel untergliedert. Nach einer Einführung in das juristische Arbeiten und einem sehr knappen historischen Rückblick (von der Armenpflege zum SGB VIII) folgen: Allgemeine Vorschriften, Jugendamt als staatlicher Wächter, Allgemeine Förderung, Förderung der Erziehung in der Familie, Kindertageseinrichtungen und Kindertagespflege, Hilfe zur Erziehung, Eingliederungshilfe, Hilfe für junge Volljährige, Mitwirkung in Gerichtsverfahren, Vormundschaft, Pflegschaft und Beistandschaft, Beurkundungsfunktionen des Jugendamts, Gesamtverantwortung und Jugendhilfeplanung, Organisation des Jugendamts, Sozialdatenschutz, Zuständigkeit, Kostenerstattung und Kostenbeteiligung. Damit werden die wesentlichen Inhalte des SGB VIII behandelt, mit Ausnahme zahlreicher Vorschriften aus dem Dritten Kapitel (Andere Aufgaben) sowie dem Fünften bis Elften Kapitel (insbesondere: Finanzierungsvorschriften), die allerdings in einem Einführungswerk wie diesem nicht unbedingt erläutert werden müssen.

Die textlichen Ausführungen werden durch Praxishinweise ergänzt. Das Werk soll Studierende der Sozialen Arbeit auf eine Tätigkeit in Jugendämtern und freier Jugendhilfe vorbereiten und zugleich eine Hilfe für Praktikerlnnen sein (Vorwort, 5).

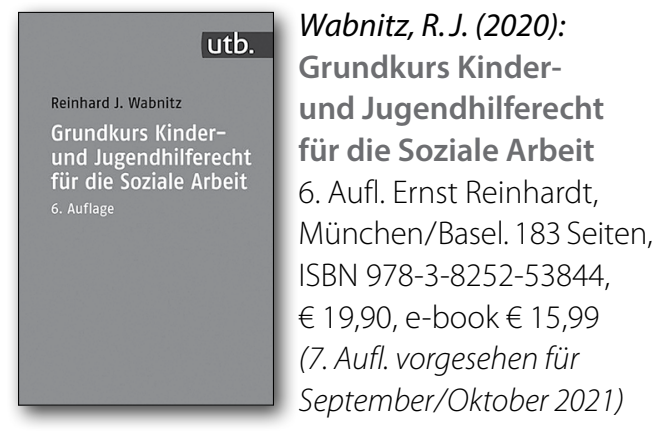

Der Autor dieses erstmals 2007 erschienenen und nunmehr in 6. Auflage 2020 vorliegenden Buches, Prof. Dr. Dr. Reinhard J. Wabnitz, ist
Professor für Rechtswissenschaft, insbesondere Familien- und Jugendhilferecht, an der Hochschule RheinMain in Wiesbaden, Ministerialdirektor a.D. und zugleich Autor dieser Sammelrezension. Dies führt zu einem gewissen Dilemma, denn einerseits soll dieses Buch hier nicht fehlen, andererseits verbieten sich „lobende Bemerkungen“; insoweit kann allerdings auf die sehr wohlwollenden Besprechungen zu Vorauflagen von Dieter Kreft in UJ 11 + 12/2010, 503 - 504, und UJ 6/2013, 283f, verwiesen werden.

Das Buch ist entsprechend den in der Regel zur Verfügung stehenden bis zu 28 Semesterwochenstunden in 14 Kapitel gegliedert: Grundsätze und Strukturprinzipien des Kinder- und Jugendhilferechts I und II; Aufgaben der Kinder- und Jugendhilfe; Förderung der Erziehung in der Familie; Jugendarbeit, Jugendsozialarbeit, Kinder- und Jugendschutz; Förderung von Kindern in Tageseinrichtungen und Kindertagespflege; Hilfe zur Erziehung (und verwandte Leistungen) I, II und III; Andere Aufgaben der Kinder- und Jugendhilfe I und II; Träger der öffentlichen Jugendhilfe und Jugendbehörden; Zusammenarbeit zwischen der öffentlichen und der freien Jugendhilfe; Verfahrensfragen und ergänzende Vorschriften. Jedes Kapitel folgt demselben Aufbau: textliche Darstellung, (insgesamt 65) grafische Übersichten und Tabellen über das „Wichtigste“ mit Erläuterungen, 14 Fallbeispiele am Ende der jeweiligen Kapitel (mit Musterlösungen am Ende des Buches) sowie zahlreiche „Vertiefungen" (ohne „Klausurrelevanz") und weiterführende Literaturhinweise.

Das Werk, das aus Lehrveranstaltungen in Wiesbaden hervorgegangen ist, will gezielt auf die Abschlussprüfung zur Lehrveranstaltung „Kinder- und Jugendhilferecht" vorbereiten, jedoch zugleich Neugierde wecken und Freude vermitteln beim Einstieg in eine für die Soziale Arbeit außerordentlich wichtige und spannende, weil lebens- und praxisnahe Rechtsmaterie. 


\section{Fazit}

Im Bereich des Kinder- und Jugendhilferechts gibt es eine ganze Reihe einschlägiger Lehr- und Lernbücher speziell für die Soziale Arbeit. Von daher besteht durchaus die "Qual der Wahl", da Interessierte sich regelmäßig nicht mehrere Werke kaufen werden. Aber potenzielle Nutzerinnen und Nutzer können auch „, beruhigt werden": fast alle hier vorgestellten Werke - mit Ausnahme des von Röchling/Schäfer - behandeln die meisten zentralen Themen des Kinderund Jugendhilferechts nach dem SGB VIII.

Wer als Studierende oder Studierender der Sozialen Arbeit lediglich "minimalistisch" die übliche Klausurprüfung im Bachelor-Grundstudium bestehen möchte, für die/den dürften die Werke von Bernzen, Schäfer, Schleicher, Schmidt und Wabnitz eine gute Hilfestellung geben. Die vorzüglichen Bücher von Kunkel und Münder/ Trenczek/von Boetticher/Tammen sind nach meinen Erfahrungen mit Blick auf die meisten Studierenden der Sozialen Arbeit (zumindest im Grundstudium) allerdings zu umfangreich und detailliert. Praktikerinnen und Praktiker sind, je nachdem, wie tief sie sich mit dem Kinder- und Jugendhilferecht befassen wollen, mit allen angezeigten Werken "gut bedient".

Prof. Dr. jur. Dr. phil.

Reinhard Joachim Wabnitz

Hochschule RheinMain, Wiesbaden

Fachbereich Sozialwesen

E-Mail: reinhard.wabnitz@hs-rm.de 